\title{
In situ surface-enhanced Raman scattering shows ligand-enhanced hot electron harvesting on silver, gold, and copper nanoparticles
}

\section{A R T I C L E I N F O}

\section{Article history:}

Received 18 October 2019

Revised 29 December 2019

Accepted 9 January 2020

\section{Keywords:}

Ligands

Hot electrons

SERS

p-Nitrothiophenol

p-Aminothiophenol

\begin{abstract}
A B S T R A C T
Hot carriers (electrons and holes) generated from the decay of localized surface plasmon resonances can take a major role in catalytic reactions on metal nanoparticles. By obtaining surface enhanced Raman scattering (SERS) spectra of p-aminothiophenol as product of the reduction of p-nitrothiophenol by hot electrons, different catalytic activity is revealed here for nanoparticles of silver, gold, and copper. As a main finding, a series of different ligands, comprising halide and non-halide species, are found to enhance product formation in the reduction reaction on nanoparticles of all three metals. A comparison with the standard electrode potentials of the metals with and without the ligands and SERS data obtained at different electrode potential indicate that the higher catalytic activity can be associated with a higher Fermi level, thereby resulting in an improved efficiency of hot carrier generation. The concept of such a ligandenhanced hot electron reduction provides a way to make light-to-chemical energy conversion more efficient due to improved electron harvesting.
\end{abstract}

(c) 2020 Elsevier Inc. All rights reserved.

\section{Introduction}

Localized surface plasmon resonances (LSPR) of metal nanostructures are widely utilized in different fields, including optics [1,2], chemistry [3-6], medicine [7], and environmental and food science [8]. In catalysis research, the application of LSPR in visible light harvesting attracts increasing attention [3-6,9]. One way of light harvesting using LSPR is based on the utilization of hot carriers, i.e., hot electrons and holes generated in the non-radiative decay of the LSPR [10-12]. Plasmonic catalysis involving hot electrons includes applications in water splitting [13], $\mathrm{CO}_{2}$ reduction [14], $\mathrm{H}_{2}$ dissociation [15], and $\mathrm{N}_{2}$ dissociation [16]. Due to such promising use, the mechanisms of plasmonic catalysis, and specifically the role of the hot carriers must be better understood. As a typical model reaction, the photo-induced reduction of $p$-nitrothiophenol (PNTP) on metal nanoparticles has frequently been used to study the role of hot electrons in plasmon-catalyzed reaction [17-22]. Normally, the plasmon-catalyzed reduction for each

* Corresponding author at: Department of Chemistry, Humboldt-Universität zu Berlin, Brook-Taylor-Str. 2, 12489 Berlin, Germany.

E-mail address: janina.kneipp@chemie.hu-berlin.de (J. Kneipp). molecule of PNTP on metal nanoparticles is a four-electronreduction, with the product 4,4'-dimercaptoazobenzene (DMAB) [17-21]. Another reaction, the six-electron-reduction of PNTP on metal nanoparticles to $p$-aminothiophenol (PATP) usually needs the addition of strong reducing agents, such as sodium borohydride [23]. As was discussed recently [24], due to a low efficiency of hot electron generation in most cases, it is hard to achieve the sixelectron-reduction process of PNTP by direct transfer of hot electrons.

Surface enhanced Raman scattering (SERS), a vibrational spectroscopic approach that exploits LSPR for the generation of high local optical fields, has been a method-of-choice for studying plasmonic catalysis, owing to its high sensitivity and surface-probing abilities [17-19,21-23,25-31]. The local sensitivity of SERS can be used to detect surface reactants, products, and intermediates $[20,23]$. Using SERS, it was observed that the six-electronreduction reaction of PNTP to PATP by hot electrons can happen on silver nanoparticles, when the presence of halides and protons is controlled, due to an improved efficiency of hot electron generation $[22,32]$. Since the six-electron-reduction by hot electrons was found only on silver nanoparticles and not on gold nanoparticles, the photo-induced recycling of silver atoms from photosensitive silver halides was proposed as a crucial step in the reaction 
mechanism [22]. As will be discussed here, it is possible to achieve the six-electron-reduction of PNTP on gold and copper nanoparticles in the absence of photosensitive halide species as well, when conditions are optimized. Specifically, we find that it can take place on gold, silver, and copper nanoparticles in the presence of protons and appropriate ligands, including $\mathrm{Cl}^{-}, \mathrm{Br}^{-}, \mathrm{I}^{-}, \mathrm{SCN}^{-}$, and $\mathrm{SC}\left(\mathrm{NH}_{2}\right)_{2}$. Monitoring the efficiency of the reaction by in situ SERS using both immobilized nanoparticles and nanoparticles in solution, it is, furthermore, demonstrated that the six-electron-reduction can also happen, albeit with lower efficiency, on silver and copper nanoparticles in the absence of any ligands, indicating that the ligands can be considered promoters instead of a mandatory element in the reaction. Comparing the reaction activities for different nanoparticles and reaction conditions, and studying the influence of defined surface potential on the reaction, we infer on the role of the ligands in hot electron induced reduction reactions. Based on comparison with standard redox potentials, we suggest that the reaction happens preferably at lower redox potential, and that this is regulated by the ligands. Nucleophile and electrophile ligands have the ability to tune the electronic properties of metal nanoparticles [33-36]. Two effects contribute to the shift of the Fermi level of metal nanoparticles: (i) the charging effect, e.g., upon the adsorption of a negatively charged nucleophile that raises the Fermi level and (ii) the chemisorption of neutral dipoles that modifies the surface potential and alters the Fermi level $[33,35]$. In our discussion we argue that the adsorption of the ligands to the metal nanoparticles alters the Fermi level and thereby influences hot electron generation. The observations reported here broaden our concept of how hot electrons can contribute to catalyzed reactions and provide new suggestions for multiple electron harvesting [3] from plasmonic catalysts.

\section{Experimental section}

\subsection{Materials}

The following chemicals (all purchased from Sigma-Aldrich) were used in the experiments: $\mathrm{HAuCl}_{4} \cdot\left(\mathrm{H}_{2} \mathrm{O}\right)_{3}, \mathrm{AgNO}_{3}, \mathrm{NaCl}, \mathrm{NaBr}$, $\mathrm{KI}, \mathrm{NaSCN}, \mathrm{SC}\left(\mathrm{NH}_{2}\right)_{2}, \mathrm{H}_{2} \mathrm{SO}_{4}, \mathrm{HCl}$, trisodium citrate, $p$ nitrothiophenol, 4-nitrobenzyl thiol. All chemicals were analytical reagent grade or better.

\subsection{Preparation of gold, silver and copper nanoparticles}

Gold nanoparticles were synthesized by citrate reduction of tetrachloroauric(III) acid according to a protocol by Lee and Meisel [37]. Typically, $300 \mu \mathrm{L}$ of tetrachloroauric(III) acid (0.25 M) was dissolved into $200 \mathrm{~mL}$ pure water and the solution is heated to boiling. $4 \mathrm{~mL}$ sodium citrate $(1 \% \mathrm{w} / \mathrm{w})$ was added into the boiling solution with further heating for $30 \mathrm{~min}$.

Silver nanoparticles were prepared by citrate reduction of silver nitrate [37]. $45 \mathrm{mg}$ of silver nitrate is dissolved into $250 \mathrm{~mL}$ pure water and the solution is head to boiling. $5 \mathrm{~mL}$ sodium citrate $(1 \% \mathrm{w} / \mathrm{w})$ was added into the boiling solution with further heating for $30 \mathrm{~min}$.

Copper nanoparticles $(25 \mathrm{~nm})$ were purchased as powder from Sigma-Aldrich.

\subsection{Preparation of immobilized metal nanoparticles}

The immobilized gold and silver nanoparticles were attached to glass slides by 3-aminopropyltriethoxysilane as previously reported [38,39]. The immobilized copper nanoparticles were prepared by drying $40 \mu \mathrm{L}$ copper nanoparticle suspension droplet (mixture of $0.1 \mathrm{~g}$ copper nanoparticles and $10 \mathrm{~mL}$ water) on glass slides. The experiment was performed by immersion of the nanostructured surfaces in $100 \mu \mathrm{M}$ PNTP $(2 \mathrm{~mL})$ for $1 \mathrm{~h}$. After washing with deionized water, the immobilized nanostructure was kept at room temperature. Then, the functionalized nanoparticle substrates were used for the Raman experiments.

\subsection{Raman experiments}

Raman spectra of the samples were measured under a Raman microscope (LabRamHR, Horiba, Jobin-Yvon, France) with a $60 \times$ water immersion objective, using different excitation wavelengths including $633 \mathrm{~nm}, 785 \mathrm{~nm}$, and $532 \mathrm{~nm}$. For the SERS measurement, a droplet of aqueous solution was placed on the immobilized nanoparticles and a $60 \times$ water immersion objective provided the focal volume from which the SERS signal was collected. The time-dependent spectra on immobilized nanoparticles after irradiation with laser light of different intensities and wavelengths were obtained with $0.1 \mathrm{~s}$ acquisition time for each, using the same laser intensity for irradiation and excitation of the Raman scattering. The SERS spectra of silver nanoparticle solution were recorded with $1 \mathrm{~s}$ acquisition time for each spectrum, using $633 \mathrm{~nm}$ as excitation laser.

For in-situ Raman / electrochemical measurements, a twoelectrode system was combined inside a cell, with conductive silicone-crystals functionalized with gold nanoparticles as working electrode surface facing towards a window of the cell. The open circuit voltage was $-0.55 \mathrm{~V}$. Raman spectra were measured using a $10 \times$ objective, using an excitation wavelength of $633 \mathrm{~nm}$. For each potential, time-dependent SERS spectra were measured after irradiation with a laser intensity of $15 \mathrm{~kW} / \mathrm{cm}^{2}$ with $5 \mathrm{~s}$ acquisition time for each spectrum, using the same laser intensity also for excitation of the Raman scattering.

\subsection{XPS measurements}

$20 \mu \mathrm{M}$ PNTP was added to $1000 \mu \mathrm{L}$ of silver nanoparticles. After incubation for $30 \mathrm{~min}, 0.1 \mathrm{M} \mathrm{HCl}$ was added into the solution. The solution was centrifuged three times at $3500 \mathrm{rpm}$ for $15 \mathrm{~min}$ to remove the $\mathrm{HCl}$ in the suspension. The final sediment was resuspended in $50 \mu \mathrm{L}$ water. A $5 \mu \mathrm{L}$ drop was applied onto a conductive indium-tin-oxide (ITO)/glass substrate. The prepared samples were dried in a vacuum chamber before XPS measurements for at least $24 \mathrm{~h}$. As control, samples using PNTP-functionalized silver nanoparticles solution without the addition of $\mathrm{HCl}$ were prepared in the same way. XPS measurements were performed using nonmonochromatized $\mathrm{Mg} \mathrm{K} \mathrm{K}_{\alpha}$ excitation (15 kV, $20 \mathrm{~mA}$ - Prevac RS 40B1) and a Scienta Omicron Argus CU electron analyzer. The XPS analysis chamber had a base pressure of $2 \times 10^{-9}$ mbar.

\section{Results and discussion}

\subsection{Six-electron reduction of PNTP on different metal nanoparticles} and in the absence of halide ions

All experiments were carried out using immobilized [38,39] Au, $\mathrm{Ag}$, and $\mathrm{Cu}$ nanoparticles on glass (Fig. $1 \mathrm{a}, \mathrm{b}$ and $\mathrm{c}$ ). The scanning electron micrographs show the formation of nanoaggregates, consistent with the red-shifted plasmon band in the absorbance spectra (Fig. S1a and S1b). The appearance of the Cu plasmon band at $580 \mathrm{~nm}$ in the absorbance spectra of the copper nanoparticles (Fig. S1b) clearly indicates that, although oxidation leads to $\mathrm{Cu} @ \mathrm{Cu}_{2} \mathrm{O}$ nanostructures (Fig. S1c) $[40,41]$, the acidic environment in all our experiments reconstitutes metallic copper [40]. Color changes visibly from black to red when the acid is added (Fig. S1b). 
a
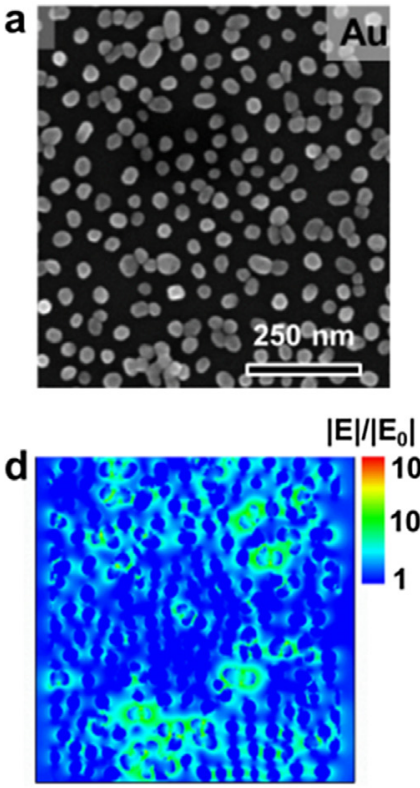

$\left(|E| /\left|E_{0}\right|\right)_{\max }=43$ b

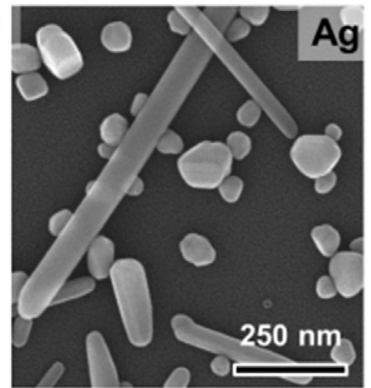

$|\mathrm{E}| /\left|\mathrm{E}_{0}\right|$

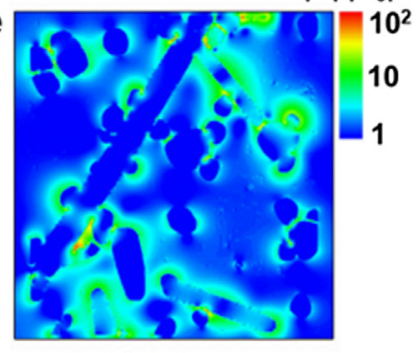

$\left(|E| /\left|E_{0}\right|\right)_{\max }=88$
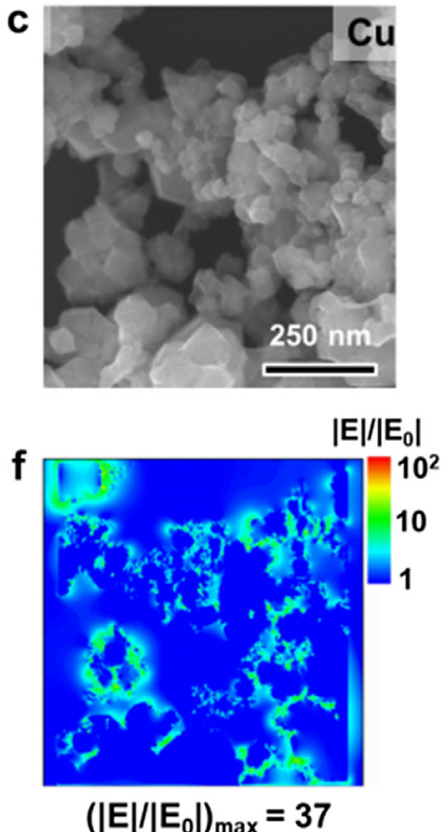

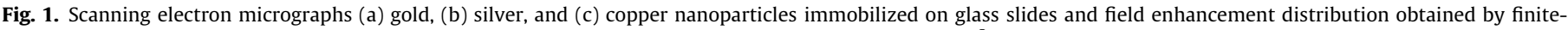

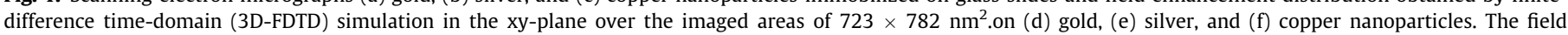
distribution in (d-f) was calculated for an excitation wavelength of $633 \mathrm{~nm}$, corresponding to the wavelength used in the experiments.

The results of finite-difference time-domain (FDTD) simulations (Fig. 1d, e, and f) indicate that the nanoaggregates provide high local optical fields, expected to lead to strong SERS enhancement [42] as well as to an efficient generation of hot electrons in the hot spots that are present $[10,43,44]$. Under excitation with light of a wavelength of $633 \mathrm{~nm}$, used in the experiments here, the local field on the nanoparticles is enhanced by up to $\sim 2$ orders of magnitude (Fig. 1d, e, and f), corresponding to maximum enhancement factors for SERS of $1.9 \times 10^{6}, 3.4 \times 10^{6}$, and $6.0 \times 10^{7}$ for copper, gold, and silver nanostructures, respectively.

Using the different immobilized metal nanostructures functionalized with PNTP, we obtained SERS spectra in the presence of $\mathrm{HCl}$ and $\mathrm{H}_{2} \mathrm{SO}_{4}$, respectively (Fig. 2). In the spectra measured in the presence of $0.1 \mathrm{M} \mathrm{HCl}$ (Fig. 2a), a signal at $1595 \mathrm{~cm}^{-1}$ assigned to a C-C vibration of the phenyl ring of PATP $[21,22,45]$ arises on silver and copper nanoparticles, accompanied by the decrease of the typical PNTP band at $1342 \mathrm{~cm}^{-1}$ that is assigned to the molecule's $\mathrm{N}-\mathrm{O}$ vibration $[17,21,22,45]$, indicating the reduction of PNTP and the formation of PATP. Hence, the $\mathrm{I}_{1595} / \mathrm{I}_{1342}$ intensity ratio can be used as an indicator to compare the catalytic activity. For $\mathrm{Cl}^{-}$this approach was corroborated by X-ray photoelectron spectroscopy (XPS, see Fig. S2 and discussion in the SI). On gold nanoparticles, PATP is formed only in the presence of $1.0 \mathrm{M} \mathrm{HCl}$ (Fig. 2a, bottom trace). The observation of PATP formation on silver nanostructures in the presence of chloride ions is in accord with previous reports $[22,32]$. The spectrum obtained with copper nanoparticles, as well as the one with the gold nanoparticles at higher $\mathrm{HCl}$ concentration in Fig. 2a indicates that the reaction also takes place on copper and gold nanoparticles. The latter is in contrast to the results reported in Refs. [22,32], where a reaction on gold nanoparticles was not observed.

As evidenced by the data in Fig. 2b, PATP is also formed on $\mathrm{Cu}$ and $\mathrm{Ag}$ nanoparticles in the presence of $0.1 \mathrm{M} \mathrm{H}_{2} \mathrm{SO}_{4}$. This means that the six-electron reduction can occur also in the absence of $\mathrm{Cl}^{-}$. The fact that the reaction takes place in the absence of halides (in $\mathrm{H}_{2} \mathrm{SO}_{4}$ ) on copper and silver nanoparticles (Fig. 2b) as well as with $\mathrm{HCl}$ on copper and on gold nanoparticles (Fig. 2a) shows that no photosensitive halide species is necessary for the six-electronreduction to take place.

The intensity ratio of the band at $1595 \mathrm{~cm}^{-1}$, assigned to PATP, and the band at $1342 \mathrm{~cm}^{-1}$, assigned to PNTP, as an indicator of the catalytic activity is compared in Fig. 2c for different nanoparticles and in the presence and absence of $\mathrm{Cl}^{-}$. At the same $\mathrm{pH}$, the relative PATP intensity found for the reaction taking place in the presence of $\mathrm{HCl}$ is higher than in $\mathrm{H}_{2} \mathrm{SO}_{4}$ (Fig. 2c), suggesting that the presence of $\mathrm{Cl}^{-}$ions enhances the reaction on all the metal nanoparticles that were used. As the chemisorption of a negatively charged nucleophile can raise the Fermi levels of the metal [33,35], here, we argue that the enhancement in the presence of the $\mathrm{Cl}^{-}$ions can be attributed to such an increase of the Fermi level, which would facilitate the generation of hot electrons.

The position of the Fermi levels with respect to the vacuum level relates to the redox potential $[33,46,47]$. Therefore, a more negative redox potential, corresponding to a higher Fermi level, will indicate a decreased confinement of electrons. This is in good agreement with the trends in the standard redox potentials of the three metals in the presence and absence of $\mathrm{Cl}^{-}$ions. Each of the standard redox potentials is smaller in the presence of $\mathrm{Cl}^{-}$than in the absence of $\mathrm{Cl}^{-}$ions: $\left(E^{0}(\mathrm{CuCl} / \mathrm{Cu})=0.12 \mathrm{~V}\right.$ is smaller than $E^{0}\left(\mathrm{Cu}^{+} / \mathrm{Cu}\right)=0.521 \mathrm{~V}, E^{0}(\mathrm{AgCl} / \mathrm{Ag})=0.222 \mathrm{~V}$ is smaller than $E^{0}\left(\mathrm{Ag}^{+} / \mathrm{Ag}\right)=0.799 \mathrm{~V}$, and $E^{0}(\mathrm{AuCl} / \mathrm{Au})=0.94 \mathrm{~V}$ is smaller than $\left.E^{0}\left(\mathrm{Au}^{+} / \mathrm{Au}\right)=1.692 \mathrm{~V}\right)$. It should be noted that the redox potential of metal nanoparticles decreases dramatically when their size is smaller than $10 \mathrm{~nm}[48,49]$, however, the nanoparticles used here are $\sim 30 \mathrm{~nm}$ or slightly larger in size, therefore possible effects of size on potential can be neglected. Although the experiments in this work were not conducted under standard conditions, the comparison of the standard redox potentials appears to provide a rationale for the discussion of the reaction activity, due to their relation with the Fermi level.

Fig. $2 \mathrm{c}$ also shows that the relative PATP intensity follows the trend $\mathrm{Cu}>\mathrm{Ag}>\mathrm{Au}$ in the presence of both, $\mathrm{HCl}$ and $\mathrm{H}_{2} \mathrm{SO}_{4}$. This is inversely related to the standard redox potential of the respective metal (see the trend of above values). Although this 

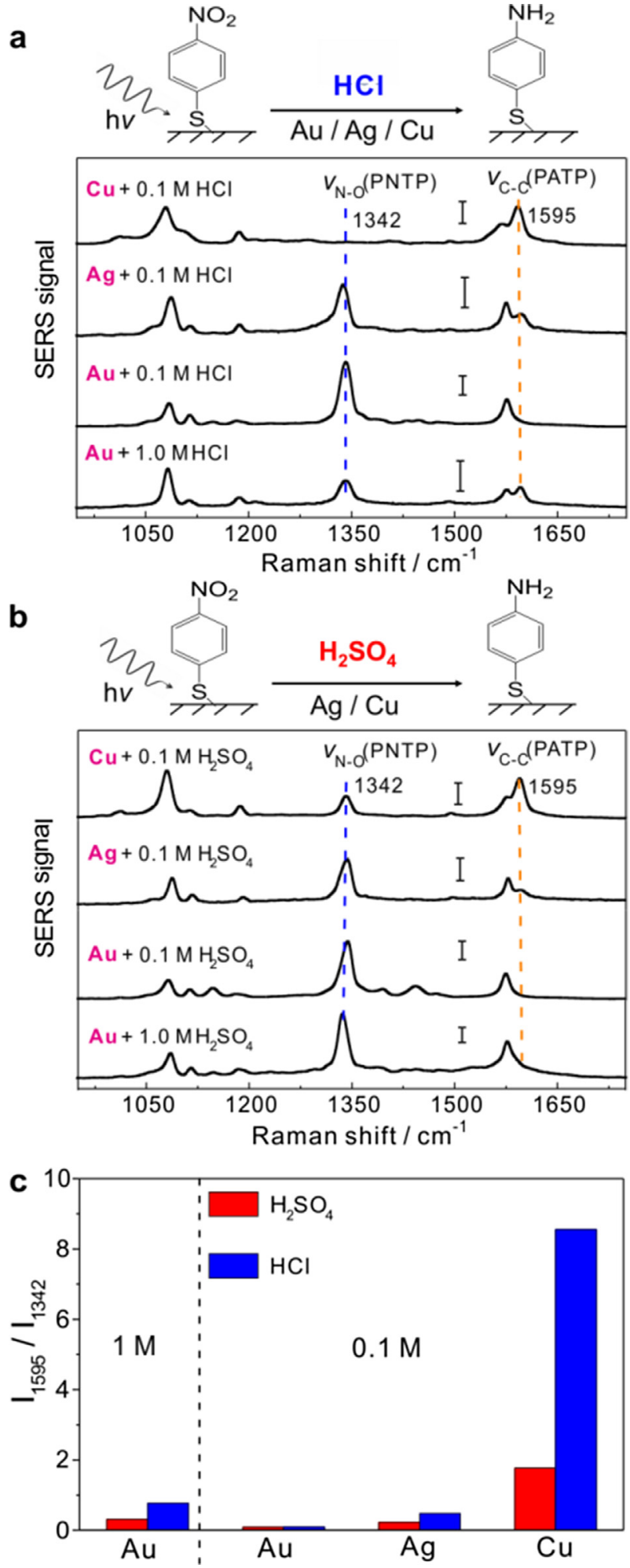

Fig. 2. The formation of the reaction product PATP on gold, silver, and copper nanoparticles is enhanced in the presence of $\mathrm{HCl}$. SERS spectra of immobilized nanoparticles functionalized with PNTP in the presence of (a) $\mathrm{HCl}$ and (b) $\mathrm{H}_{2} \mathrm{SO}_{4}$ after irradiation for $60 \mathrm{~s}$, and (c) the corresponding intensity ratio $\mathrm{I}_{1595} / \mathrm{I}_{1342}$ of the band at $1595 \mathrm{~cm}^{-1}$ assigned to PATP and the band at $1342 \mathrm{~cm}^{-1}$ assigned to PNTP. Scale bars in (a) from top to bottom: $5000 \mathrm{cps}, 2000 \mathrm{cps}, 8000 \mathrm{cps}, 4000 \mathrm{cps}$. Scale bars in (b) from top to bottom: $8000 \mathrm{cps}, 2000 \mathrm{cps}, 16,000 \mathrm{cps}, 2000 \mathrm{cps}$. ( $\lambda_{\text {excitation }}$ $633 \mathrm{~nm}$; acquisition time $0.1 \mathrm{~s}$; laser intensity $50 \mathrm{~kW} / \mathrm{cm}^{2}$ ).

relationship may also be determined by the different confinement of the electrons, other factors, such as nanoparticle size, shape and aggregate formation, cannot be excluded.
In agreement with this discussion, in situ Raman experiments in an electrochemical cell reveal a more efficient photo-reduction of PNTP under more negative potential (higher Fermi level) $[33,46]$ on silver nanoparticles (Fig. S3). This is also consistent with a previous report about a potential-dependent photoreaction of nitrobenzene derivatives [50].

The inverse relation of the reaction efficiency and electrode potential dominates over other effects that could relate to catalytic activity as well. These include, e.g., the highest local field enhancement in nanoparticle aggregates (compare Fig. 1d-e), which would positively influence the generation of hot electrons [10,51,52] specifically on silver, or the affinity of each respective metal for $\mathrm{Cl}^{-}$ions, which is higher in gold compared to silver and copper [53], that could inhibit electron-hole recombination [22] and yield higher PATP signals for the gold nanoparticles. Neither of these effects were observed though when comparing product formation.

\subsection{Six-electron reduction is favored under high laser intensity}

We performed the PNTP-to-PATP reaction on all nanoparticles and in the presence of $\mathrm{HCl}$ and $\mathrm{H}_{2} \mathrm{SO}_{4}$, respectively, for different irradiation intensities using a laser wavelength of $633 \mathrm{~nm}$. On gold nanoparticles, the reaction was monitored using other Raman excitation (and irradiation) wavelengths as well (Fig. S4), but it yielded much lower PATP signals, in accord with the fact that they did not match the observed LSPR absorbance maxima (Fig. S1) and in agreement with previous reports [27,29]. Fig. 3 displays the intensity ratio $\mathrm{I}_{1595} / \mathrm{I}_{1342}$ as measure of the relative amount of the reaction product for different irradiation times (Fig. 4). The corresponding spectra are shown in Fig. S5. In all samples, except for the gold nanoparticles in $\mathrm{H}_{2} \mathrm{SO}_{4}$ in the absence of $\mathrm{Cl}^{-}$ (Fig. 3a), higher relative PATP signals are found when the irradiation intensities increase (Fig. 3b-f). At intensities around 5 $\mathrm{kW} / \mathrm{cm}^{2}$, the low $\mathrm{I}_{1595} / \mathrm{I}_{1342}$ ratio indicates that the reaction hardly occurs at all on gold and silver nanoparticles (Fig. 3a-d, black traces). This is also the case for copper nanoparticles in the absence of $\mathrm{Cl}^{-}$(Fig. 3e). Different from this, in the presence of $\mathrm{HCl}$, also low irradiation intensities lead to formation of the reaction product on copper nanoparticles (Fig. 3f). At irradiation intensities of 50 $\mathrm{kW} / \mathrm{cm}^{2}$ or higher, the intensity ratio rises much faster on both silver and copper nanoparticles when $\mathrm{Cl}^{-}$ions from $\mathrm{HCl}$ are present (compare Fig. $3 \mathrm{c}$ with $\mathrm{d}$ and Fig. 3e with $\mathrm{f}$, respectively). In contrast, for gold nanoparticles the presence of $\mathrm{HCl}$ is crucial for the reaction to occur, also when the laser intensity is increased to $125 \mathrm{~kW} / \mathrm{cm}^{2}$ (compare Fig. 3a and b).

All the results demonstrate that a high laser intensity of a specific wavelength promotes the six-electron-reduction of PNTP to PATP, which confirms its nature as a photo-catalytic reaction that is influenced by the LSPR. The generated hot electrons are in a dynamic equilibrium, and recombine with holes [54]. An increase of laser intensity can improve the number of hot carriers, but will also increase the local temperature on the nanoparticle surfaces, which could enhance the reaction rate even further.

A higher catalytic activity at higher intensity is also found in the reduction of other nitrobenzene derivatives. On copper nanoparticles, 4-nitrobenzyl thiol is reduced by hot electrons to form 4-aminobenzyl thiol as reaction product (Fig. S6).

\subsection{Ligands enhance hot electron reduction}

The possibility to observe the six-electron reduction on gold nanoparticles in the presence of $\mathrm{HCl}$ (Fig. 2a and c), and the faster product formation when $\mathrm{HCl}$ (Fig. 3f) is present on the copper nanoparticles necessitates a discussion of the role of the $\mathrm{Cl}^{-}$ions. Fig. 4a and $\mathrm{b}$ shows the SERS spectra of PNTP using gold and copper nanoparticles with excitation of $633 \mathrm{~nm}$ before and after the 

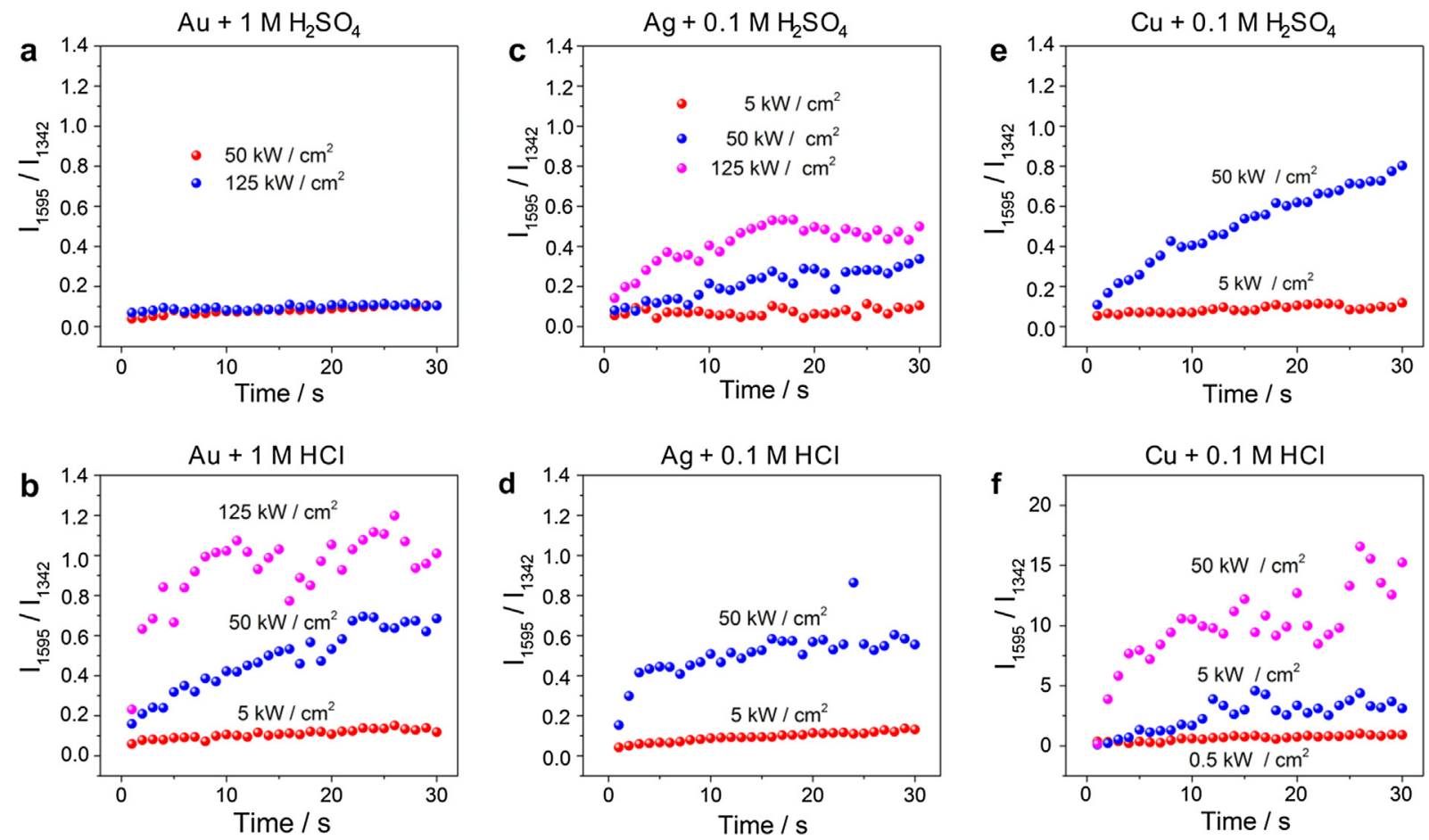

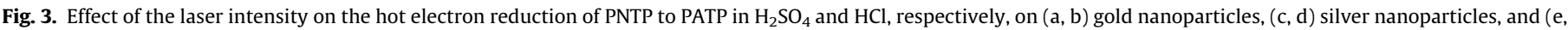

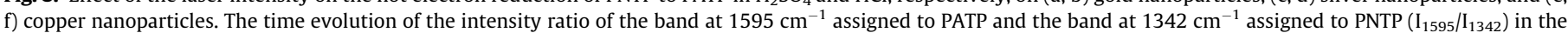

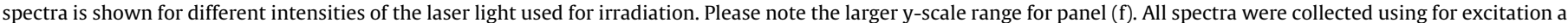
laser wavelength of $633 \mathrm{~nm}$ at the indicated intensities; acquisition time per spectrum was $0.1 \mathrm{~s}$.
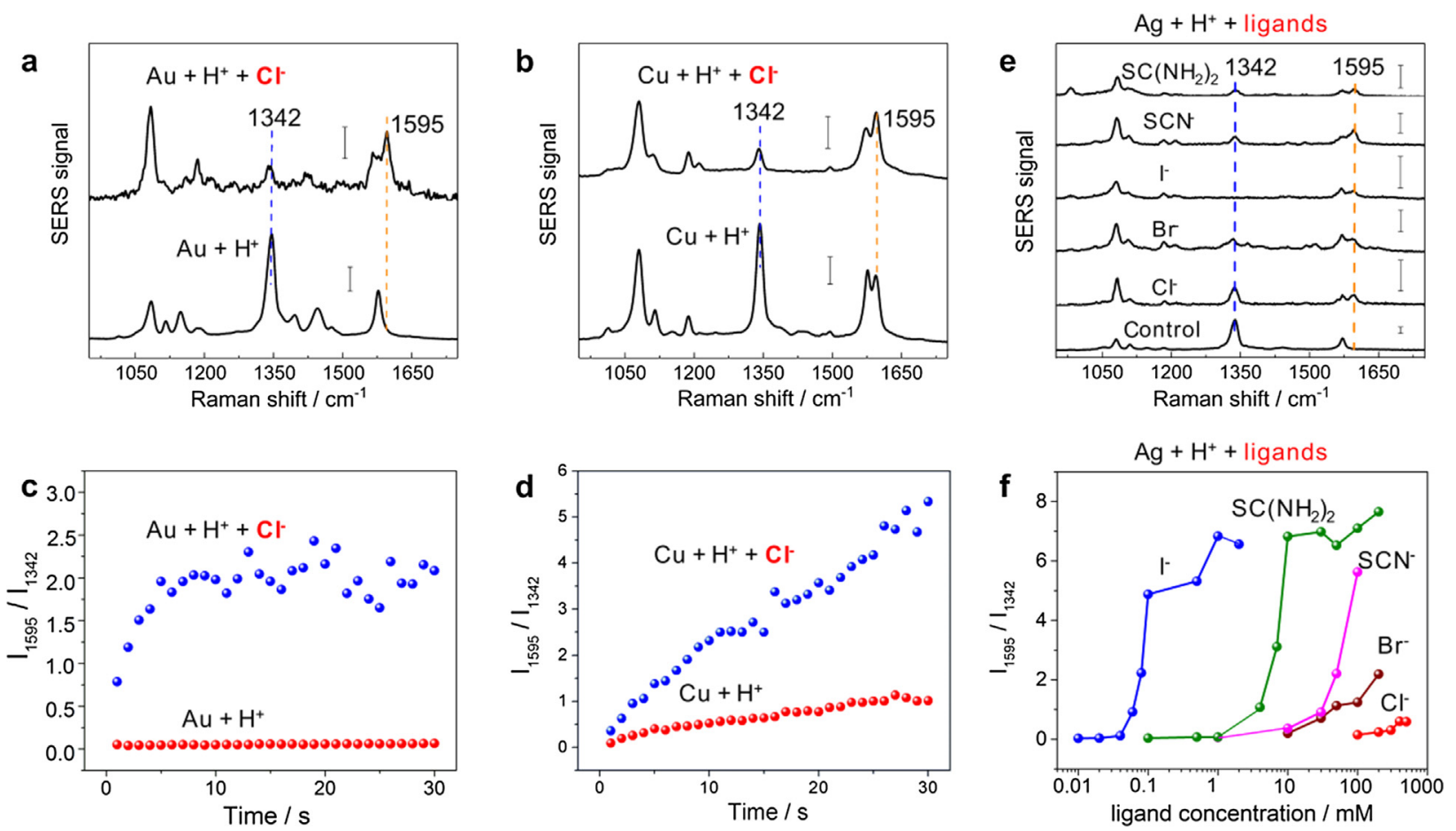

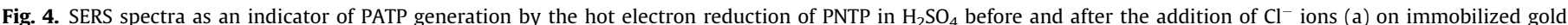

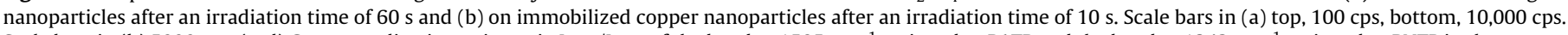

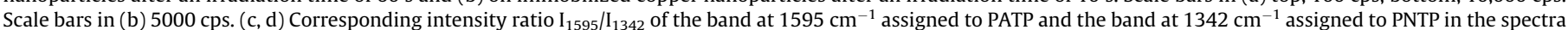

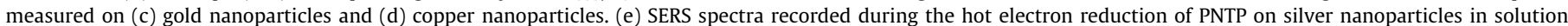

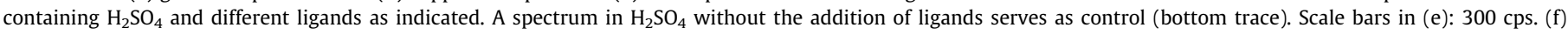

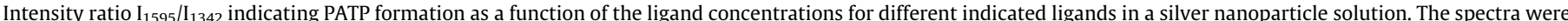

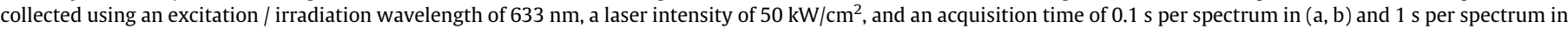


addition of $\mathrm{Cl}^{-}$ions from $\mathrm{NaCl}$ in $\mathrm{H}_{2} \mathrm{SO}_{4}$. The PATP signals are stronger with the addition of $\mathrm{Cl}^{-}$ions than in $\mathrm{H}_{2} \mathrm{SO}_{4}$ alone, and the rise of the relative PATP signal $\mathrm{I}_{1595} / \mathrm{I}_{1342}$ is faster (Fig. $4 \mathrm{c}$ and $\mathrm{d}$ ). The results indicate that the $\mathrm{Cl}^{-}$ions enhance the hot electron reduction on both gold and copper nanoparticles. Since photosensitive halide species are not formed on gold and copper, we infer that this must take place without the formation of a photosensitive species that has been suggested for silver nanoparticles [22].

In accordance with previous work [22], the spectra obtained with silver nanoparticles in the presence of $\mathrm{Cl}^{-}, \mathrm{Br}^{-}$and $\mathrm{I}^{-}$ions (Fig. 4e) show an increase in the relative PATP signal visualized by the ratio $\mathrm{I}_{1595} / \mathrm{I}_{1342}$ (Fig. $4 \mathrm{f}$, bottom four traces). Interestingly, this occurs also with the addition of the pseudo-halide ligands $\mathrm{SCN}^{-}$and $\mathrm{SC}\left(\mathrm{NH}_{2}\right)_{2}$ (Fig. 4f, upper two traces). The effect of the concentration of all ligands on the relative PATP signal is shown in Fig. 4f. The onset of the ratio $\mathrm{I}_{1595} / \mathrm{I}_{1342}$ (increase in the relative PATP signal) is at very low concentrations for $\mathrm{I}^{-}$and at highest concentrations for $\mathrm{Cl}^{-}$(Fig. 4f), and has a good correlation with the standard electrode potentials of the corresponding metal-ligand systems [53], including the non-halide ligands: $E^{0}(\mathrm{AgI} / \mathrm{Ag})=-0.1$ $52 \mathrm{~V}$ is smaller than $E^{0}\left(\mathrm{Ag}\left(\mathrm{SC}\left(\mathrm{NH}_{2}\right)_{2}\right)_{4}^{+} / \mathrm{Ag}\right)=-0.013 \mathrm{~V}, E^{0}(\mathrm{AgSCN} /$ $\mathrm{Ag})=0.0895 \mathrm{~V}$ is very similar to $E^{0}(\mathrm{AgBr} / \mathrm{Ag})=0.07133 \mathrm{~V}$, and both are smaller than $E^{0}(\mathrm{AgCl} / \mathrm{Ag})=0.222 \mathrm{~V}$. The largest value is found for $E^{0}\left(\mathrm{Ag}_{2} \mathrm{SO}_{4} / \mathrm{Ag}\right)=0.654 \mathrm{~V}$. The observation of lower potentials for the $\mathrm{I}^{-}$species and also for $\mathrm{SC}\left(\mathrm{NH}_{2}\right)_{2}$ and their positive influence on activity are in accordance with the conclusion drawn above that the ligands must increase the Fermi level and hence decrease the confinement of the electrons.

Also at neutral $\mathrm{pH}$, where a four-electron-reduction process with the product DMAB takes place due to the deficiency of protons [17-19,21,55], the $\mathrm{Cl}^{-}$and $\mathrm{Br}^{-}$ions enhance the photoinduced reduction of PNTP on the gold and silver nanoparticles (Fig. S7). This demonstrates that the ligands enhance both the six-electron and the four-electron reduction by hot carriers under both acidic and neutral condition, respectively.

\subsection{Proposed mechanism of the hot electron-induced reduction of PNTP}

As indicated by the results above, the hot electron induced reduction of PNTP to PATP is influenced by the metal species, the ligands, and the intensity of the light that excites the LSPR. Using the description for the generation of hot electrons from Refs. [12,54,56,57] shown in picture (i) of Scheme 1, we infer from our data that the six-electron-reduction of PNTP on metal nanoparticles can take place as follows: In the absence of the ligand, the required energy $\emptyset$ for the generation of hot electrons is high (Scheme 1, picture (i)), only very few electrons are energetic enough to participate in the reduction reaction at the nanoparticle surface, and the reaction is slow. When the ligand adsorbs on the nanoparticle surface (picture (ii) in Scheme 1), the Fermi level with respect to vacuum level on the nanoparticle surface increases (and modifies the surface potential) [33-36], and the required energy $\emptyset$ will decrease. As a result, more energetic hot electrons are generated. This description is different from the recent model which involves a surface field mediated excitation of hybridized (interfacial) metal-molecule states formed upon chemisorption [57]. Specifically, under acidic conditions in the absence of ligands, the photo-induced reduction of PNTP is slow due to the smaller number of hot electrons. In contrast, in the presence of the ligand, the decay of the LSPR produces more hot electrons, leading to the fast reduction of PNTP to PATP. The proposed mechanism is supported by the observation of a higher reaction efficiency at more negative potentials shown in the in-situ Raman / electrochemical experiments (Fig. S3). In addition, XPS data clearly showed the

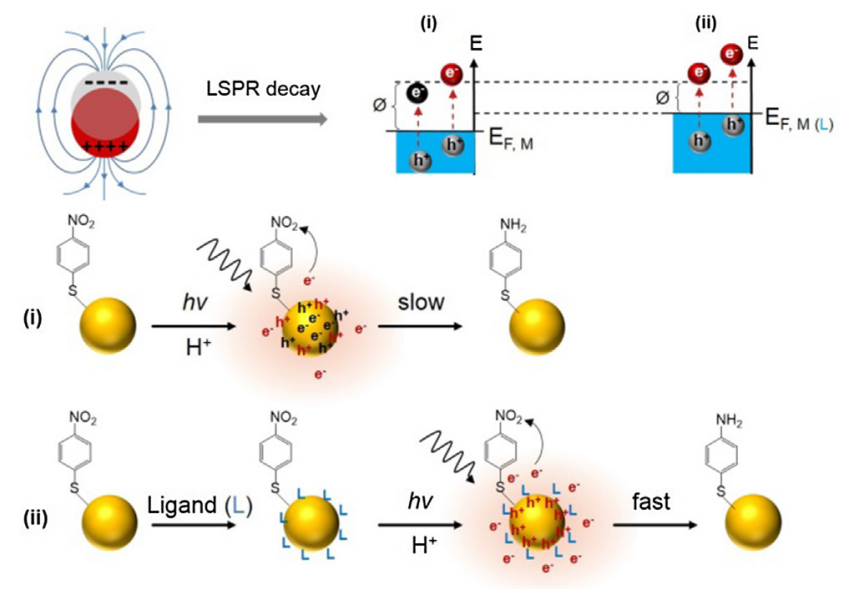

Scheme 1. Schematic illustration of the ligand-enhanced hot electron reduction on metal nanoparticles. Generation of hot electron-hole pairs within the metal from the decay of the localized surface plasmon resonance (LSPR) in (i) the absence and (ii) the presence of a ligand and the proposed mechanism for the six-electronreduction reduction of PNTP to PATP on the metal nanoparticles under acidic condition for both situations.

presence of $\mathrm{Cl}^{-}$after treatment of the PNTP decorated $\mathrm{Ag}$ particles with $\mathrm{HCl}$ and a possible correlation to the formation of PATP (Fig. S2).

The ligands may also have further roles, including the impairment of charge-carrier recombination [22,58], directly serving as electron donor [59], the change of LSPR band width that is related to the chemical induced damping (CID) process [10], and formation of a photosensitive metal-ligand complex as has been discussed in the case of silver nanoparticles [22]. The data discussed here, therefore, extend the previous discussion [22] of the mechanism of the six-electron reduction as follows:

$\mathrm{M}+\mathrm{L} \rightarrow \mathrm{M}(\mathrm{L})$

$\mathrm{M}(\mathrm{L})+\mathrm{h} v \rightarrow \mathrm{M}^{+}(\mathrm{L})+\mathrm{e}^{-}$

PNTP $+6 \mathrm{e}^{-}+6 \mathrm{H}^{+} \rightarrow$ PATP $+2 \mathrm{H}_{2} \mathrm{O}$

$\mathrm{M}$ and $\mathrm{L}$ in the above reaction equations stand for the metal and the ligand, respectively. The adsorption of ligands on the nanoparticles (1) is followed by the generation of the hot electrons when the LSPR decays (2), and by the reduction of PNTP by these energetic electrons (3). While the concentration of protons will influence whether a four-electron process or the six-electron process takes place as step (3), the metal species, laser intensity, and ligands determine the hot electron density in step (2) and hence the overall reaction rate. In this step, the role of the ligands is to decrease the energy threshold and to limit the electron-hole recombination, both of which increase the hot electron density on the nanoparticle surface. To better understand the interaction of the ligands with the nanoparticle surfaces, other vibrational spectroscopies with high sensitivity to probe molecular species at interfaces $[60,61]$ could provide help in the future.

The counterpart half reactions can happen as discussed previously [59,62]. As described in Eqs. (4), [13,3](5) and (6), [59] water and ligands (e.g., $\mathrm{Cl}^{-}, \mathrm{Br}^{-}, \mathrm{I}^{-}$) can act as electron donors in the counterpart half reactions.

$$
\begin{aligned}
& 4 \mathrm{M}^{+}+2 \mathrm{H}_{2} \mathrm{O} \rightarrow 4 \mathrm{M}+\mathrm{O}_{2}+4 \mathrm{H}^{+} \\
& \mathrm{M}^{+}+\mathrm{H}_{2} \mathrm{O} \rightarrow \mathrm{M}+\mathrm{OH}+\mathrm{H}^{+} \\
& \mathrm{M}^{+}+\mathrm{L}^{-} \rightarrow \mathrm{L}+\mathrm{M}
\end{aligned}
$$


The observation of the hot-electron reduction of 4-nitrobenzyl thiol on copper nanoparticles (Fig. S6), where charge transfer may be hampered by the additional carbon in the chain, suggests that in step (3) there possibly occurs a direct transfer of hot electrons from the metal to the $\mathrm{NO}_{2}$ group of PNTP. However, as suggested in previous work, hot electrons could also be injected into water via the plasmon excitation [63]. Therefore, a direct contact of $\mathrm{NO}_{2}$ to the metal surface would not be mandatory to achieve the electron transfer process.

\section{Conclusion}

From the reported data it can be concluded that the hot electron reduction on metal nanoparticles is enhanced by decreasing the energy threshold for the electrons to become hot, and by limiting electron-hole recombination. Both effects are achieved when ligands that increase the Fermi level are present at the metal surface. This finding broadens the concept of the hot electron induced reduction on metal nanoparticles. It further provides suggestions for several applications of hot electrons in real life, including the utilization of copper nanoparticles for plasmonic catalysis and their application in important reactions, e.g., for the synthesis of nitrobenzene derivatives in the production of pharmaceuticals, dyes, and related compounds [64].

\section{Declaration of Competing Interest}

The authors declare that they have no known competing financial interests or personal relationships that could have appeared to influence the work reported in this paper.

\section{Acknowledgements}

We thank Dr. Ulrich Gernert (ZELMI, TU Berlin) for support regarding scanning electron microscopy, Dr. Virginia Merk (HU), Dr. Merwe Buurman, and Prof. Dr. Ulrich Panne (BAM) for fruitful discussion. Financial support of the research by DFG GSC 1013 SALSA (fellowship to Z.Z.), ERC grant no 259432 (J.K.) and DFG FOR 2177 InCheM (J.K.) is gratefully acknowledged.

\section{Appendix A. Supplementary material}

Supplementary data to this article can be found online at https://doi.org/10.1016/j.jcat.2020.01.006.

\section{References}

[1] F. Benz, M.K. Schmidt, A. Dreismann, R. Chikkaraddy, Y. Zhang, A. Demetriadou, C. Carnegie, H. Ohadi, B. de Nijs, R. Esteban, Science 354 (2016) 726-729.

[2] L.M. Liz-Marzán, C.J. Murphy, J. Wang, Chem. Soc. Rev. 43 (2014) 3820-3822.

[3] Y. Kim, J.G. Smith, P.K. Jain, Nat. Chem. 10 (2018) 763-769.

[4] L. Zhou, D.F. Swearer, C. Zhang, H. Robatjazi, H. Zhao, L. Henderson, L. Dong, P. Christopher, E.A. Carter, P. Nordlander, Science 362 (2018) 69-72.

[5] E. Kazuma, J. Jung, H. Ueba, M. Trenary, Y. Kim, Science 360 (2018) 521-526.

[6] U. Aslam, S. Chavez, S. Linic, Nat. Nanotechnol. 12 (2017) 1000-1005.

[7] E.C. Dreaden, A.M. Alkilany, X. Huang, C.J. Murphy, M.A. El-Sayed, Chem. Soc. Rev. 41 (2012) 2740-2779.

[8] K. Saha, S.S. Agasti, C. Kim, X. Li, V.M. Rotello, Chem. Rev. 112 (2012) 27392779.

[9] Z. Zhang, C. Zhang, H. Zheng, H. Xu, Acc. Chem. Res. 52 (2019) 2506-2515.

[10] G.V. Hartland, L.V. Besteiro, P. Johns, A.O. Govorov, ACS Energy Lett. 2 (2017) $1641-1653$.

[11] A.V. Zayats, S. Maier, Adv. Opt. Mater. 5 (2017) 1700508.
[12] M.L. Brongersma, N.J. Halas, P. Nordlander, Nat. Nanotechnol. 10 (2015) 25-38.

[13] J. Lee, S. Mubeen, X. Ji, G.D. Stucky, M. Moskovits, Nano Lett. 12 (2012) 5014 5019.

[14] S. Yu, A.J. Wilson, J. Heo, P.K. Jain, Nano Lett. 18 (2018) 2189-2194.

[15] L. Zhou, C. Zhang, M.J. McClain, A. Manjavacas, C.M. Krauter, S. Tian, F. Berg, H. O. Everitt, E.A. Carter, P. Nordlander, Nano Lett. 16 (2016) 1478-1484.

[16] J.M.P. Martirez, E.A. Carter, J. Am. Chem. Soc. 139 (2017) 4390-4398.

[17] B. Dong, Y. Fang, L. Xia, H. Xu, M. Sun, J. Raman Spectrosc. 42 (2011) 12051206.

[18] E.M. van Schrojenstein Lantman, T. Deckert-Gaudig, A.J. Mank, V. Deckert, B.M. Weckhuysen, Nat. Nanotechnol. 7 (2012) 583-586.

[19] L.-B. Zhao, M. Zhang, Y.-F. Huang, C.T. Williams, D.-Y. Wu, B. Ren, Z.-Q. Tian, J. Phys. Chem. Lett. 5 (2014) 1259-1266.

[20] H.-K. Choi, K.S. Lee, H.-H. Shin, Z.H. Kim, J. Phys. Chem. Lett. 7 (2016) 40994104.

[21] H.-K. Choi, W.-H. Park, C.-G. Park, H.-H. Shin, K.S. Lee, Z.H. Kim, J. Am. Chem. Soc. 138 (2016) 4673-4684.

[22] W. Xie, S. Schlücker, Nat. Commun. 6 (2015) 7570.

[23] Z. Zhang, U. Gernert, R. Gerhardt, E.-M. Höhn, D. Belder, J. Kneipp, ACS Catal. 8 (2018) 2443-2449.

[24] K. Wu, J. Chen, J.R. McBride, T. Lian, Science 349 (2015) 632-635.

[25] Y. Huang, Y. Fang, Z. Yang, M. Sun, J. Phys. Chem. C 114 (2010) 18263-18269.

[26] Y.-F. Huang, H.-P. Zhu, G.-K. Liu, D.-Y. Wu, B. Ren, Z.-Q. Tian, J. Am. Chem. Soc. 132 (2010) 9244-9246.

[27] Z. Zhang, V. Merk, A. Hermanns, W.E. Unger, J. Kneipp, ACS Cata. 7 (2017) $7803-7809$.

[28] H. Zhang, X.-G. Zhang, J. Wei, C. Wang, S. Chen, H.-L. Sun, Y.-H. Wang, B.-H. Chen, Z.-L. Yang, D.-Y. Wu, J. Am. Chem. Soc. 139 (2017) 10339-10346.

[29] Y.F. Huang, M. Zhang, L.B. Zhao, J.M. Feng, D.Y. Wu, B. Ren, Z.Q. Tian, Angew. Chem. 53 (2014) 2353-2357.

[30] K. Xu, R. Zhou, K. Takei, M. Hong, Adv. Sci. (2019) 1900925.

[31] Z. Zhang, T. Deckert-Gaudig, V. Deckert, Analyst 140 (2015) 4325-4335.

[32] E. Cortés, W. Xie, J. Cambiasso, A.S. Jermyn, R. Sundararaman, P. Narang, S. Schlücker, S.A. Maier, Nat. Commun. 8 (2017) 14880.

[33] M.D. Scanlon, P. Peljo, M.A. Méndez, E. Smirnov, H.H. Girault, Chem. Sci. 6 (2015) 2705-2720

[34] A. Henglein, J. Phys. Chem. 97 (1993) 5457-5471.

[35] P. Mulvaney, T. Linnert, A. Henglein, J. Phys. Chem. 95 (1991) 7843-7846.

[36] E. Smirnov, M.D. Scanlon, D. Momotenko, H. Vrubel, M.A. Méndez, P.-F. Brevet, H.H. Girault, ACS Nano 8 (2014) 9471-9481.

[37] P. Lee, D. Meisel, J. Phys. Chem. 86 (1982) 3391-3395.

[38] V. Joseph, M. Gensler, S. Seifert, U. Gernert, J.r.P. Rabe, J. Kneipp, J. Phys. Chem. C 116 (2012) 6859-6865.

[39] V. Joseph, C. Engelbrekt, J. Zhang, U. Gernert, J. Ulstrup, J. Kneipp, Angew. Chem. 51 (2012) 7592-7596.

[40] G.H. Chan, J. Zhao, E.M. Hicks, G.C. Schatz, R.P. Van Duyne, Nano Lett. 7 (2007) 1947-1952.

[41] X. Guo, C. Hao, G. Jin, H.Y. Zhu, X.Y. Guo, Angew. Chem. 53 (2014) 1973-1977.

[42] K. Kneipp, H. Kneipp, J. Kneipp, Acc. Chem. Res. 39 (2006) 443-450.

[43] P. Christopher, H. Xin, A. Marimuthu, S. Linic, Nat. Mater. 11 (2012) 1044.

[44] A. Sousa-Castillo, M. Comesaña-Hermo, B. Rodriguez-Gonzalez, M.s. PérezLorenzo, Z. Wang, X.-T. Kong, A.O. Govorov, M.A. Correa-Duarte, J. Phys. Chem. C 120 (2016) 11690-11699.

[45] W. Xie, B. Walkenfort, S. Schlücker, J. Am. Chem. Soc. 135 (2012) 1657-1660.

[46] H. Reiss, J. Phys. Chem. 89 (1985) 3783-3791.

[47] L.M. Peter, Photocatalysis: fundamentals and perspectives, Royal Soc. Chem. (2016) 1-28

[48] O.S. Ivanova, F.P. Zamborini, J. Am. Chem. Soc. 132 (2010) 70-72.

[49] O.S. Ivanova, F.P. Zamborini, Anal. Chem. 82 (2010) 5844-5850.

[50] S. Sun, R.L. Birke, J.R. Lombardi, K.P. Leung, A.Z. Genack, J. Phys. Chem. 92 (1988) 5965-5972.

[51] H. Zhang, A.O. Govorov, J. Phys. Chem. C 118 (2014) 7606-7614.

[52] L.V. Besteiro, A.O. Govorov, J. Phys. Chem. C 120 (2016) 19329-19339.

[53] J.G. Speight, Lange's Handbook of Chemistry, McGraw-Hill, New York, 2005.

[54] C. Clavero, Nat. Photonics 8 (2014) 95-103.

[55] K. Kim, J.-Y. Choi, K.S. Shin, J. Phys. Chem. C 119 (2015) 5187-5194.

[56] J.B. Khurgin, Nat. Nanotechnol. 10 (2015) 2-6.

[57] C. Boerigter, U. Aslam, S. Linic, ACS Nano 10 (2016) 6108-6115.

[58] B. Kafle, M. Poveda, T.G. Habteyes, J. Phys. Chem. Lett. 8 (2017) 890-894.

[59] Y. Tian, T. Tatsuma, J. Am. Chem. Soc. 127 (2005) 7632-7637.

[60] Y. Tong, I.Y. Zhang, R.K. Campen, Nat. Commun. 9 (2018) 1313.

[61] F. Yesudas, M. Mero, J. Kneipp, Z. Heiner, J. Chem. Phys. 148 (2018), 104702.

[62] H. Shi, G. Li, H. Sun, T. An, H. Zhao, P.-K. Wong, Appl. Catal., B. 158 (2014) $301-$ 307.

[63] P. Zilio, M. Dipalo, F. Tantussi, G.C. Messina, F. De Angelis, Light Sci. Appl. 6 (2017) e17002.

[64] J. Feng, S. Handa, F. Gallou, B.H. Lipshutz, Angew. Chem. 55 (2016) 8979-8983. 